\title{
Hepatic metastasis from bile duct cancer of the ampulla is effectively controlled by multidisciplinary treatment including S-1, gemcitabine and bevacizumab: A case report
}

\author{
YUKIHIKO TOKUNAGA ${ }^{1}$, TOSHIHIKO KAWASAKI ${ }^{2}$, HIROKAZU SASAKI $^{1}$, \\ SHIGEKI MATSUEDA ${ }^{2}$ and TOHRU SAITO ${ }^{1}$ \\ Departments of ${ }^{1}$ Surgery, and ${ }^{2}$ Gastroenterology, Osaka North Japan Post Hospital, Osaka 530-8798, Japan
}

Received June 1, 2009; Accepted September 2, 2009

DOI: 10.3892/ol_00000084

\begin{abstract}
The liver is the most common site for recurrent metastases from bile duct cancer (BDC) in the ampullary area. However, the optimal chemotherapy regimen for recurrent hepatic metastases has not yet been established. An oral combined fluoropyrimidine drug, S-1 (tegafur, gimeracil and oteracil), has recently been introduced alone or in combination with gemcitabine for BDC. A 67-year-old man underwent a pancreaticoduodenectomy (PD) for early stage distal BDC in the ampullary area. A small hepatic metastasis developed 8 months after the PD. Combined chemotherapy of S-1 $\left(80 \mathrm{mg} / \mathrm{m}^{2}\right)$ and gemcitabine $\left(1000 \mathrm{mg} / \mathrm{m}^{2}\right)$ was started after radiofrequency ablation (RFA) of the hepatic tumor. Although complete response was achieved and maintained for 4 months with chemotherapy, there was regrowth of the tumor. We performed hepatic segmentectomy for radical treatment. Fourteen months after the hepatectomy, metastasis developed again in the remnant liver. Bevacizumab was added to the combination chemotherapy with S-1 and gemcitabine, since the cancer seemed resistant to the chemotherapy alone. The patient has been well managed for 3 years by a multidisciplinary treatment with surgery, RFA and the combination chemotherapy on an outpatient basis. This case indicates that distal BDC even in an early stage has a more malignant potential than anticipated. The multidisciplinary treatment including surgery, RFA and combination chemotherapy of S-1, gemcitabine and bevacizumab was effective for BDC with hepatic metastasis. This chemotherapy is feasible on an outpatient basis and may be one of the treatment options for metastatic BDC.
\end{abstract}

Correspondence to: Dr Yukihiko Tokunaga, Department of Surgery, Osaka North Japan Post Hospital, 1-1-6 Nakazaki, Kita-ku, Osaka 530-8798, Japan

E-mail: tokunagay@usay6.com

Key words: bile duct cancer, S-1, gemcitabine, bevacizumab

\section{Introduction}

True adenocarcinoma of the distal bile duct (i.e. arising in the retroduodenal or intrapancreatic portion of the common bile duct) are uncommon neoplasms that comprise a small portion of all periampullary malignancies necessitating pancreaticoduodenectomy (PD) $(1,2)$. Since experience with these neoplasms is limited $(2,3)$, they are often analyzed in conjunction, either with upper- and mid-third bile duct carcinoma (4), or with periampullary malignancies in general (5).

Among several potential recently innovated oral agents, an oral combined fluoropyrimidine S-1 (tegafur, gimeracil and oteracil) has been recently used for gastrointestinal (6), pancreatic (7) and bile duct cancer (BDC) (8). Other promising agents include gemcitabine $(9,10)$ and bevacizumab (11). To date, however, few reports are available regarding the effects of a combination of these agents on metastatic BDC.

We report a case of hepatic metastasis from distal BDC of the ampulla, in which long-term control was obtained by a multidisciplinary treatment with radiofrequency ablation (RFA), surgery and chemotherapy using S-1, gemcitabine and bevacizumab.

\section{Case report}

A 65-year-old man presented with intermittent abdominal pains for several days. His complete blood count showed leukocytosis and thrombocytosis. Laboratory studies disclosed elevations in bilirubin $(2.5 \mathrm{mg} / \mathrm{dl})$, liver enzymes (AST 577 IU/l, ALT 452 IU/l) and alkaline phosphatase (149 IU/l). Ultrasonography (US) showed a slight dilatation of the common bile duct and the hepatic duct without any sign of a stone or tumor. Endoscopic retrograde cholangiopancreatogaphy (ERCP) showed a filling defect in the distal bile duct of the ampullary area, and a biopsy revealed adenocarcinoma. Computed tomography combined with angiography revealed a hypervascular tumor in the distal bile duct (Fig. 1). The patient underwent a PD in July, 2006. A surgical specimen showed a small polypoid tumor $(10 \times 6 \times 6 \mathrm{~mm})$ in the distal bile duct of the ampullary area (Fig. 2). According to TNM classification, this case was classified as T1N0M0 (stage I). Pathological 


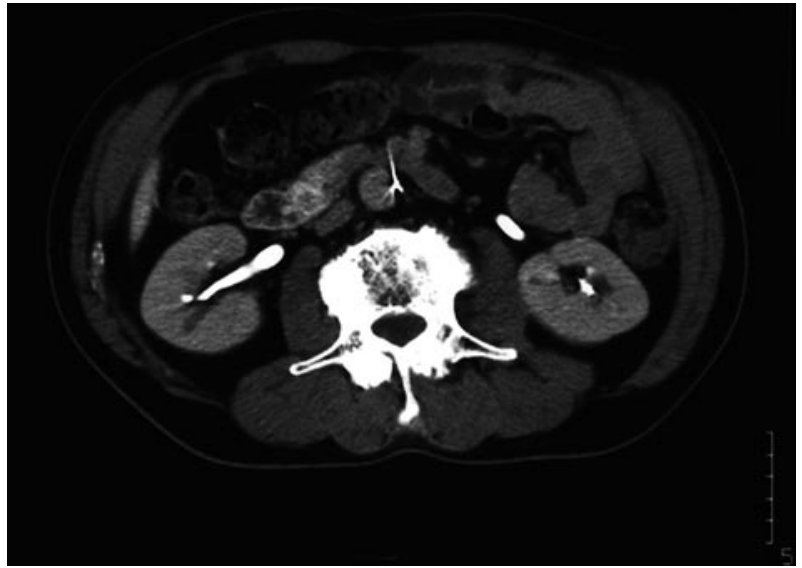

Figure 1. Computed tomography combined with angiography revealed a hypervascular tumor in the distal bile duct.

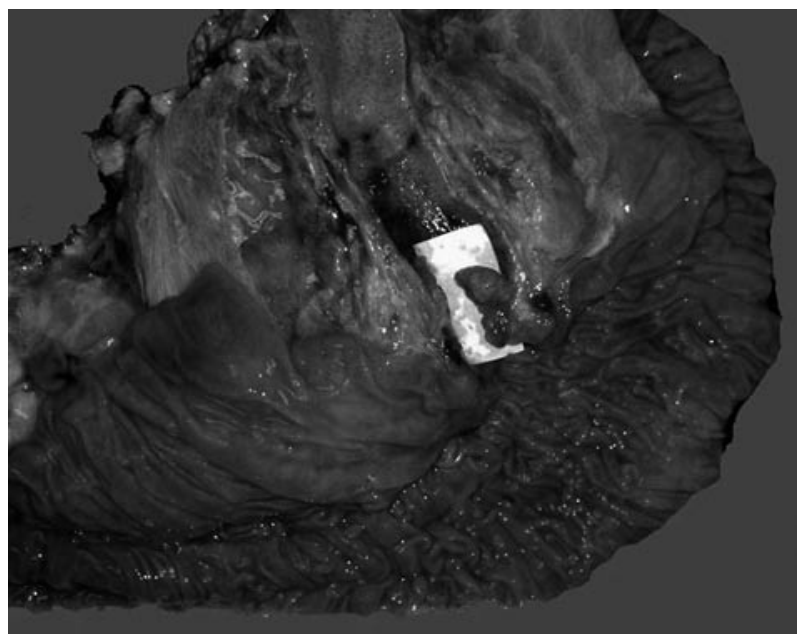

Figure 2. Surgical specimen showed a small polypoid tumor $(10 \times 6 \times 6 \mathrm{~mm})$ in the distal bile duct of the ampullary area.

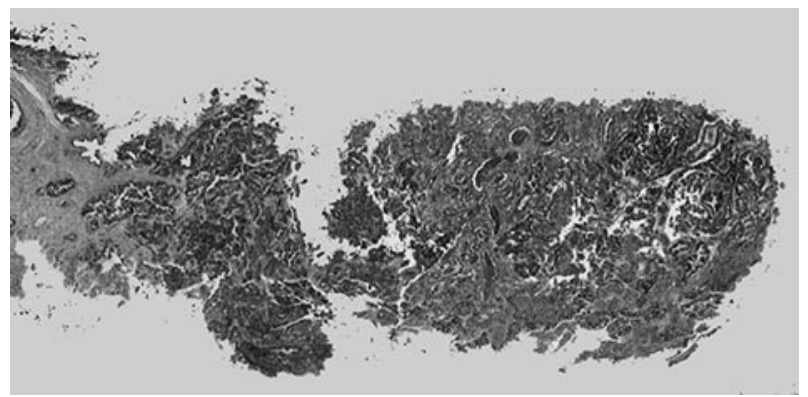

Figure 3. Pathological findings showed a papillary adenocarcinoma limited to the biliary mucosa, without any infiltration to vessels or the neural plexus.

findings revealed a papillary adenocarcinoma limited to the biliary mucosa without any infiltration to vessels or the neural plexus (Fig. 3). No metastasis was noted in the regional lymph nodes. His post-operative course was uneventful, and he was discharged from the hospital without any complications.

The patient was well without any evidence of recurrence for 7 months, being managed with adjuvant chemotherapy

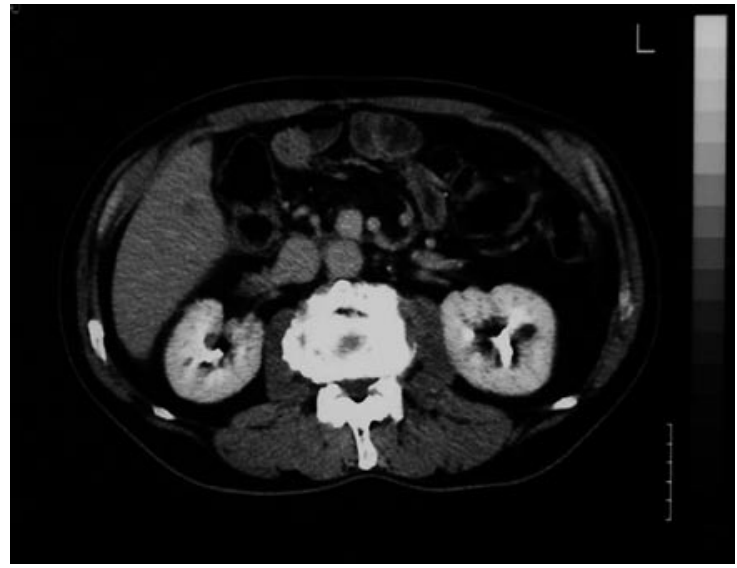

Figure 4. CT and magnetic resonance imaging showed a hepatic tumor.

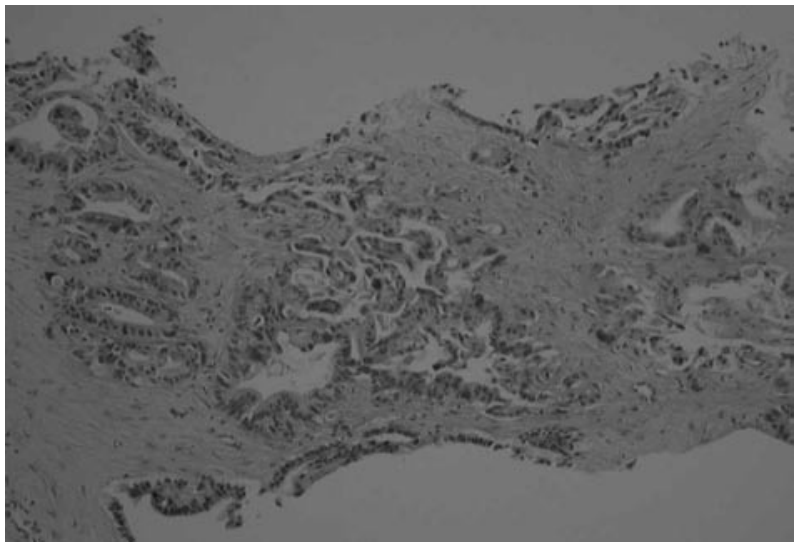

Figure 5. Pathological findings of the biopsy from the liver tumor, which showed a papillary adenocarcinoma, conjectured to be from the metastasis of the primary tumor.

using oral UFT (a combination of tegafur and uracil, $375 \mathrm{mg} /$ $\mathrm{m}^{2} /$ day). Eight months after surgery, tumor markers including carcinoembryonic antigen (CEA) and carbohydrate antigen (CA)19-9 gradually increased. CT scans and magnetic resonance imaging showed a hepatic tumor $\sim 1 \mathrm{~cm}$ in diameter (Fig. 4). A biopsy revealed a papillary adenocarcinoma conjectured to be the result of metastasis from the primary tumor (Fig. 5), and RFA was performed upon consent of the patient.

Combined chemotherapy with $\mathrm{S}-1\left(80 \mathrm{mg} / \mathrm{m}^{2}\right.$ on days $1-14$ with 7 days off) and gemcitabine $\left(1000 \mathrm{mg} / \mathrm{m}^{2}\right.$ on day 8$)$ was initiated after RFA. The chemotherapy was continued on an outpatient basis. After 3 courses of chemotherapy, CT scans revealed that the lesion resolved with a decline in the CA19-9 level (Fig. 6), and the patient appeared to achieved a complete response. Although the response was maintained for 4 months with the chemotherapy, there was regrowth of the tumor to $2 \mathrm{~cm}$ in diameter. We performed RFA again and maintained the chemotherapy upon consent of the patient. Since another metastatic lesion developed in the marginal zone 4 months after the RFA (Fig. 7), we performed hepatic segmentectomy. Fourteen months after the hepatectomy, another hepatic metastasis developed in the remnant liver. Bevacizumab $(10 \mathrm{mg} / \mathrm{kg}$ on day 8) was added to the combination chemotherapy with 


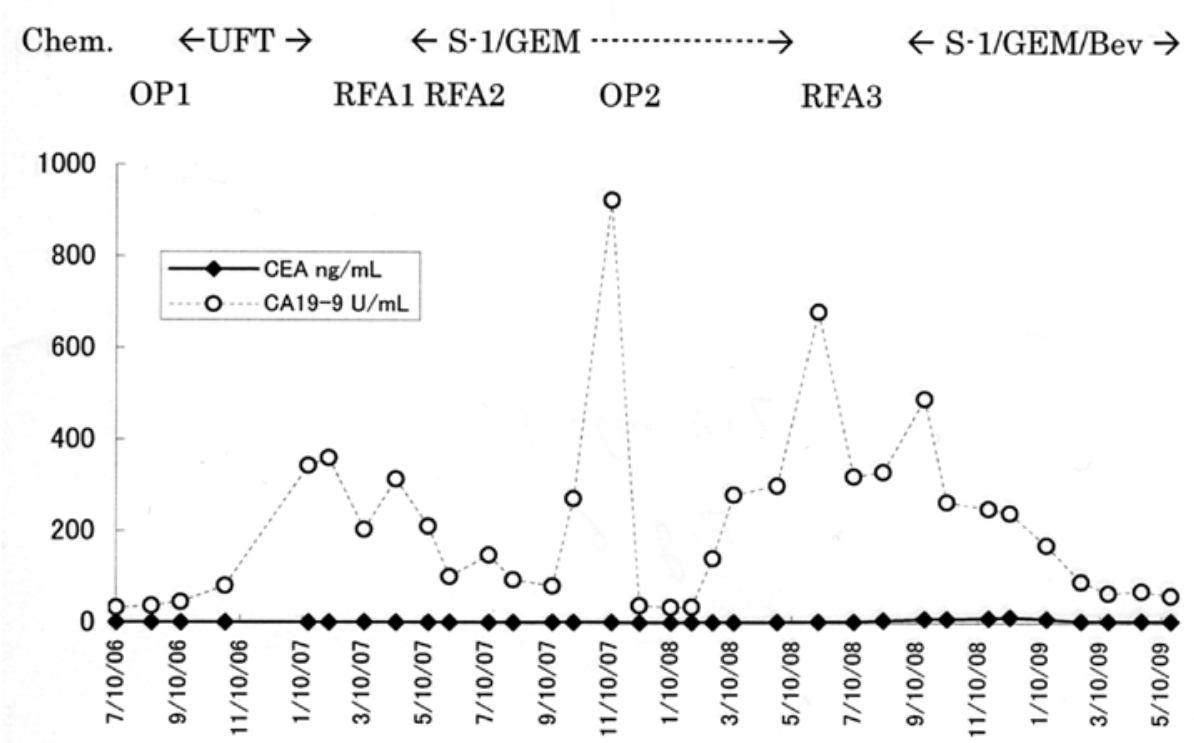

Figure 6. Time course change in carcinoembryonic antigen (CEA) and carbohydrate antigen (CA)19-9 levels. Bev, bevacizumab; GEM, gemcitabine; OP1, operation 1 (pancreaticoduodenectomy); OP2, operation 2 (hepatectomy); RFA, radiofrequency ablation; S-1, oral combined fluoropyrimidine (tegafur, gimeracil and oteracil); UFT, oral combined fluoropyrimidine (uracil and tegafur).

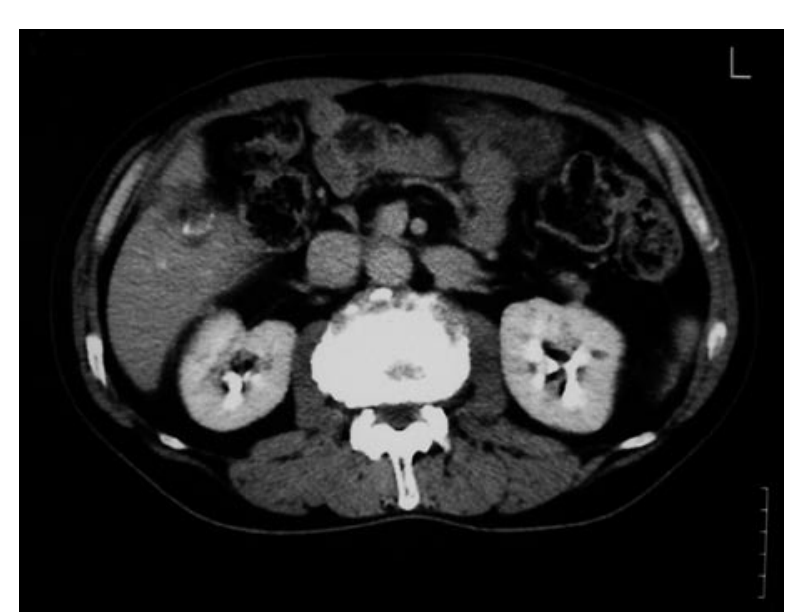

Figure 7. Hepatic segmentectomy was performed for another metastatic lesion which developed in the marginal zone 4 months after the second RFA.

S-1 and gemcitabine, since the cancer seemed resistant to the chemotherapy alone. After 2 courses of chemotherapy with bevacizumab, abdominal contrast $\mathrm{CT}$ scans revealed that the lesion was resolved with a decline in the tumor markers. The patient was effectively managed for 3 years after the initial PD by a multidisciplinary treatment with RFA, surgery and the combination chemotherapy on an outpatient basis.

\section{Discussion}

Experiences regarding the outcome of surgery, survival and management of patients with distal BDC are limited and have not been well reported for several reasons. First, it is the most infrequent type of periampullary malignancy including pancreatic, ampullary and duodenal cancers. Second, this subset of cholangiocarcinomas is usually described in combination with hilar and intrahepatic cholangiocarcinomas which appear to have different predisposing factors $(4,5)$ and, therefore, may have a very different natural history.

Todoroki et al reported recurrence patterns of ampullary cancer including distal BDC after currative resection (12). The overall frequency of distant metastasis was $44 \%$ and that of local recurrence was $11 \%$. The most frequent site of distant metastasis was the liver (67\%) and the second most common was the distant lymph nodes (54\%) followed by the peritoneum (21\%) and the lung (21\%). From the histopathologic point of view, locoregional spread to lymph nodes within the resected specimen was less common for distal BDC than for pancreatic ductal cancer $(2,13)$. In contrast to lymph nodes metastases, the incidences of direct lymphatic infiltration (27\%) and perineural invasion $(60 \%)$ suggest a more aggressive tumor biology and portend a less favorable prognosis despite the small size of the primary tumors $(2,3)$.

According to published data, the 5-year survival after resection of distal BDC is approximately $20-30 \%$ (1-3). Although these neoplasms represent a minority of the patient population undergoing PD and the experience at any institution with distal DBC is necessarily limited, similar clinical outcome and survival are consistent and suggest a somewhat better prognosis than for pancreatic ductal cancer but not quite as good as for periampullary adenocarcinomas arising from the ampullary or duodenal mucosa $(14,15)$. This case indicates that distal $\mathrm{BDC}$ even in an early stage has more malignant potential than anticipated, and that meticulous follow-up with tumor markers and imaging studies are necessary in these cases.

The optimal chemotherapy regimen for recurrent hepatic metastasis has not yet been established since previous chemotherapy with 5-fluorouracil (5-FU) has not proved beneficial in unresectable BDC and in an adjuvant setting after surgical resection $(16,17)$. New anticancer agents such as S-1 and gemcitabine, recently introduced in clinical practice, provide the potential to improve prognosis in patients with unresectable BDC and recurrent BDC after surgery (6-10). 
S-1, a new oral fluoropyrimidine, consists of tegafur and two modulators, 5-choloro-2,4-dihydroxypyrimidine (CDHP) and potassium oxonate (OXO). CDHP is a competitive inhibitor of dihydropyrimidine dehydrogenase, which is an enzyme for 5-FU degradation. OXO ameliorates gastrointestinal toxicity of tegafur by a competitive inhibition of orotate phosphoribosyltransferase in the gastrointestinal mucosa (6). As a single agent in a phase II study for advanced pancreatic cancer (7) and BDC (8), the response rate of S-1 was approximately $40 \%$, with a low incidence of grade 3 or 4 toxicity $(7,8)$. Recently, Nakamura et al reported a response rate of $48 \%$ and an overall survival of 12.5 months in a phase II trial of S-1 with gemcitabine for metastatic pancreatic cancer (18). The study of the effects of S-1 with gemcitabine on advanced BDC is limited, though reports on the effects of gemcitabine as a single agent are available (10).

Bevacizumab was added to the chemotherapy in our case since the hepatic metastases developed, as the chemotherapy was continued. This agent was proved to be quite effective for treatment of advanced colorectal cancer when used in combination with chemotherapy (11). To date, however, few reports are available regarding the effects of bevacizumab on pancreatic cancer or BDC (19).

In the present case, the hepatic metastases were effectively controlled by a multidisciplinary treatment including RFA, surgery and combination chemotherapy with S-1, gemcitabine and bevacizumab. This chemotherapy is feasible on an outpatient basis and may be one of the treatment options for metastatic BDC.

\section{References}

1. Nakeeb A, Pitt HA, Sohn TA, Coleman J, Abrams RA, Piantadosi S, Hruban RH, Lillemoe KD, Yeo CJ and Cameron JL: Cholangiocarcinoma: a spectrum of intrahepatic, perihilar and distal tumors. Ann Surg 224: 463-475, 1996.

2. Fong Y, Blumgart LH, Lin E, Fortner JG and Brennan MF: Outcome of treatment for distal bile duct cancer. Br J Surg 83: 1712-1715, 1996.

3. Bortolasi L, Burgart LJ, Tsiotos GG, Lugue-De Leon E and Sarr MG: Adenocarcinoma of the distal bile duct (a clinicopathologic outcome analysis after curative resection). Dig Surg 17: 36-41, 2000.

4. Nagorney DM, Donohue JH, Farnell MB, Schleck CD and Ilstrup DM: Outcome after curative resection of cholangiocarcinoma. Arch Surg 128: 871-879, 1993.

5. Kayahara $M$, Nagakawa $T$, Ueno $K$, Ohta $T$, Tsukioka $Y$ and Miyazaki I: Surgical strategy for carcinoma of the pancreas head area based on clinicopathologic analysis of nodal involvement and plexus invasion. Surgery 117: 616-623, 1995.

6. Shirasaka T, Shimamoto Y, Ohshimo H, Yamaguchi H, Kato T, Yonekura K and Fukushima M: Development of a novel form of an oral 5-fluorouracil derivative (S-1) directed to the potentiation of the tumor selective toxicity of 5-fluorouracil by two biochemical modulators. Anticancer Drugs 7: 548-557, 1996.
7. Okusaka T, Funakoshi A, Furuse J, Boku N, Yamao K, Ohkawa S and Saito H: A late phase II study of S-1 for metastatic pancreatic cancer. Cancer Chemother Pharmacol 61: 615-621, 2008.

8. Ueno H, Okusaka T, Ikeda M, Takezako Y and Morizane C: Phase II study of S-1 in patients with advanced biliary tract cancer. Br J Cancer 15: 1769-1774, 2004.

9. Lin MH, Chen JS, Chen HH and Su WC: A phase II trial of gemcitabine in the treatment of advanced bile duct and periampullary carcinoma. Chemotherapy 49: 154-158, 2003.

10. Okusaka T, Ishii H, Funakoshi A, Yamao K, Ohkawa S, Saiot S, Saito $\mathrm{H}$ and Tsuyuguchi T: Phase II trial of single-agent gemcitabine in patients with advanced biliary tract cancer. Cancer Chemother Pharmacol 57: 647-653, 2006.

11. Hurwitz $H$, Fehrenbacher L, Novotny $W$, Cartwright $T$, Hainsworth J, Heim W, Berlin J, Baron A, Griffing S, Holmgren E, Ferrara N, Fyfe G, Rogers B, Ross R and Kabbinavar F: Bevacizumab plus irinotecan, fluorouracil and leucovorin for metastatic colorectal cancer. N Eng J Med 350: 2335-2342, 2004.

12. Todoroki T, Koike N, Morishita Y, Kawamoto T, Ohkochi N, Shoda J, Fukuda Y and Takahashi H: Patterns and failure after curative resections of carcinoma of the ampulla of Vater. Ann Surg Oncol 10: 1176-1183, 2003.

13. Bhuiya MR, Nimura Y, Kamiya K, Kondo S, Fukata S, HayakawaN and Shionoya S: Clinicopathologic studies in perineural invasion of bile duct carcinoma. Ann Surg 215: 344-349, 1992.

14. Chan C, Herrera MF, De la Garza L, Quantanilla-Martinez L, Vargas-Vorackova F, Richaud-Patin Y, Llorente L, Uscanga L, Robles-Diaz G and Leon E: Clinical behavior and prognostic factors of periampullary adenocarcinoma. Ann Surg 222: 632-637, 1995.

15. Woo SM, Ryu JK, Lee SH, Yoo JW, Park JK, Kim YT, Jang JY, Kim SW, Kang GH and Yoon YB: Recurrence and prognostic factors of ampullary carcinoma after radical resection: comparison with distal extrahepatic cholangiocarcinoma. Ann Surg Oncol 14: 3195-3201, 2007.

16. Takada $T$, Nimura $Y$, Katoh $H$, Nagakawa $T$, Nakayama $T$, Matsushiro T, Amano $\mathrm{H}$ and Wada K: Prospective randomized trial of 5-fluorouracil, doxorubicin and mitomycin $\mathrm{C}$ for non-resectable pancreatic and biliary carcinoma: multicenter randomized trial. Hepatogastroenterology 45: 2020-2026, 1998.

17. Takada T, Amano H, Yasuda H, Nimura Y, Matsushiro T, Kato H, Nagakawa T and Nakayama T: Is postoperative adjuvant chemotherapy useful for gallbladder carcinoma? A phase III multicenter prospective randomized controlled trial in patients with resected pancreaticobiliary carcinoma. Cancer 95: 1685-1695, 2002.

18. Nakamura $K$, Yamaguchi $T$, Ishihara $T$, Sudo $K$, Kato $H$ and Saisho H: Phase II trail of oral S-1 combined with gemcitabine in metastatic pancreatic cancer. Br J Cancer 94: 1575-1579, 2006.

19. Kindler HL, Niedzwiecki D, Hollis D, Martin EW, Schilsky RL and Goldberg RM: A double-blind, placebo-controlled, randomized phase III trial of gemcitabine (G) plus bevacizumab (B) versus gemcitabine plus placebo $(\mathrm{P})$ in patients (pts) with advanced panreatic cancer (PC): a preliminary analysis of Cancer and Leukemia Group B (CALGB) 80303. ASCO Gastrointestinal Cancer Symposium: abs. 108, 2007. 